LAPTH-003/11

\title{
Phantom Black Holes and Sigma Models
}

\author{
Mustapha Azreg-Aïnou ${ }^{(a) *}$ \\ Gérard Clément ${ }^{(\mathrm{b}) \dagger}$ \\ Júlio C. Fabris ${ }^{(\mathrm{c}, \mathrm{d})}$ ‡ \\ Manuel E. Rodrigues ${ }^{(b, c) \S}$ \\ (a) Başkent University, Department of Mathematics, \\ Bağlica Campus, Ankara, Turkey \\ (b) Laboratoire de Physique Théorique LAPTH (CNRS), \\ B.P.110, F-74941 Annecy-le-Vieux cedex, France \\ (c) Universidade Federal do Espírito Santo, \\ Centro de Ciências Exatas - Departamento de Física, \\ Av. Fernando Ferrari, 514 - Campus de Goiabeiras, \\ CEP29075-910 - Vitória/ES, Brazil \\ (d) IAP, Institut d'Astrophysique de Paris, \\ 98 bis, Bd Arago - 75014 Paris, France
}

18 February 2011

\begin{abstract}
We construct static multicenter solutions of phantom Einstein-Maxwell-dilaton theory from null geodesics of the target space, leading to regular black holes without spatial symmetry for certain discrete values of the dilaton coupling constant. We also discuss the three-dimensional gravitating sigma models obtained by reduction of phantom Einstein-Maxwell, phantom Kaluza-Klein and phantom Einstein-Maxwell-dilatonaxion theories. In each case, we generate by group transformations phantom charged black hole solutions from a neutral seed.
\end{abstract}

\footnotetext{
*E-mail: azreg@baskent.edu.tr

$\dagger$ E-mail: gclement@lapp.in2p3.fr

†E-mail: fabris@pq.cnpq.br

§E-mail: esialg@gmail.com
} 


\section{Introduction}

Phantom gravitating field theories are theories where one or more of the matter fields appear in the action with a kinetic term of the "wrong sign", so that they are coupled repulsively to gravity. This implies the violation of the null energy condition $p+\rho \geq 0$. At the quantum level, such hypothetical fields could form a "ghost condensate" vacuum, which would lead to modifications of gravity in the infra-red limit [1]. Present observational evidence cannot rule out this possiblity, and even under certain conditions it seems to favor a phantom scenario. For example, the seven-year WMAP results, combined with $B A O$ and $H_{0}$ data, indicate that, imposing a constant equation of state parameter $\omega=p / \rho$, it comes out that $\omega=-1.10 \pm 0.14$ for a flat universe, and $\omega=-1.44 \pm 0.27$ for a non-flat universe [2]. Ghost condensate models may also lead to a singularity-free primordial scenario generating a perturbation spectrum in agreement with the present observations [3].

The presence of phantom fields in the action results in interesting new possibilities for stationary solutions, such as wormhole solutions in the case of a phantom scalar field [4], zero-mass black holes [5], and cold black holes with a multiply degenerate horizon and an infinite horizon area [6,7]. The static, spherically symmetric black hole solutions to Einstein-Maxwell-dilaton (EMD) theory with phantom Maxwell and/or dilaton field were systematically investigated in [8]. Nine classes of asymptotically flat phantom black holes, as well as two classes of non-asymptotically flat phantom black holes, were found, and their causal structure was analyzed, leading to a rich variety of sixteen different types of causal structures.

The purpose of the present work is to go beyond the static spherically symmetric approach of [8] by using nonlinear sigma-model techniques. Dimensional reduction of a sector of four- or higher-dimensional gravitating field theories to three dimensions leads to a gravitating sigma model (see e.g. [9,10]). Besides providing a path (essentially equivalent to that followed in [8]) to the construction of static spherically symmetric solutions as target space (or potential space) geodesics, the sigma-model approach can be used to generate new solutions in two ways. First, solutions without spatial symmetry, also called multicenter, or BPS, solutions generalizing the static Majumdar-Papapetrou solutions of Einstein-Maxwell (EM) theory [11] and their stationary counterpart [12] can be constructed from null geodesics of the target space [13, 14]. Second, in special cases the target space is a symmetric space, or coset, leading to the possibility of generating new solutions, e.g. stationary axisymmetric solutions, by applying group transformations to the coset representative of a seed solution.

In the present paper, we shall follow the two approaches just described. In the next Section, we consider the target space for static solutions of phantom EMD, and show that the null geodesics of this target space lead, for certain discrete values of the dilaton coupling constant, to regular multicenter black hole solutions. In Section 3 we review the sigma model for stationary Einstein-Maxwell theory, and construct the sigma model for stationary phantom Einstein-Maxwell $(E \bar{M})$ theory. We then consider in Section 4 the sigma model for fivedimensional Einstein theory with two timelike Killing vectors, which is equivalent to stationary phantom EMD theory for the special value $\lambda=-\sqrt{3}$ of the dilaton coupling constant. We apply the matrix method to generate a phantom charged rotating black hole solution from the Kerr solution. In Section 5, we first review and derive the properties of the cosets for Einstein-Maxwell-dilaton-axion (EMDA) and its phantom counterpart (EMDA), and again apply the sigma-model approach to generate a charged rotating black hole solution of EMDA. The last section contains our conclusions.

\section{Static phantom EMD: multicenter solutions}

We consider the string-inspired action

$$
S=-\int \mathrm{d}^{4} x \sqrt{-g}\left[\mathscr{R}-2 \eta_{1} g^{\mu v} \partial_{\mu} \phi \partial_{v} \phi+\eta_{2} \mathrm{e}^{2 \lambda \phi} F_{\mu v} F^{\mu v}\right]
$$


which describes Einstein-Maxwell-dilaton gravity, with $\lambda$ the real dilaton-Maxwell coupling constant, and $\eta_{1}$, $\eta_{2}$ the dilaton-gravity and Maxwell-gravity coupling constants. Normal EMD corresponds to $\eta_{2}=\eta_{1}=+1$, while phantom couplings of the Maxwell field $F=\mathrm{d} A$ or/and dilaton field $\phi$ are obtained for $\eta_{2}=-1$ or/and $\eta_{1}=-1$. For short, we call the corresponding theories, which include E $\bar{M} \mathrm{D}\left(\eta_{1}=+1, \eta_{2}=-1\right)$, EMD $\left(\eta_{1}=-1, \eta_{2}=+1\right)$ and $\overline{\mathrm{MD}}\left(\eta_{1}=-1, \eta_{2}=-1\right)$, phantom EMD.

EMD and its phantom derivatives are invariant under an electric-magnetic duality exchanging electric and magnetic fields and simultaneously reversing the sign of the dilaton field. For this reason, we can restrict the investigation of static solutions to the purely electric case, and parametrize the spacetime metric and the electric field by

$$
\mathrm{d} s^{2}=f \mathrm{~d} t^{2}-f^{-1} h_{i j} \mathrm{~d} x^{i} \mathrm{~d} x^{j}, \quad F_{i 0}=\frac{1}{\sqrt{2}} \partial_{i} v
$$

$(i=1,2,3)$. Then, the original four-dimensional EMD equations can be reduced to a three-dimensional problem deriving from the gravitating sigma-model action [15]

$$
S_{3}=\int \mathrm{d}^{3} x \sqrt{h}\left[R(h)-G_{A B}(\boldsymbol{X}) \partial_{i} X^{A} \partial_{j} X^{B} h^{i j}\right]
$$

where $R(h)$ is the Ricci scalar constructed from the 3-dimensional metric $h_{i j}$, and $G_{A B}(\boldsymbol{X})$ is the target space metric, with $X^{1}=f, X^{2}=v, X^{3}=\phi$,

$$
\mathrm{d} l^{2}=G_{A B} \mathrm{~d} X^{A} \mathrm{~d} X^{B}=\frac{\mathrm{d} f^{2}}{2 f^{2}}-\frac{\eta_{2}}{f} \mathrm{e}^{2 \lambda \phi} \mathrm{d} v^{2}+2 \eta_{1} \mathrm{~d} \phi^{2} .
$$

The field equations derived from (2.3) are

$$
\begin{aligned}
& R_{i j}(h)=G_{A B} \partial_{i} X^{A} \partial_{j} X^{B}, \\
& \partial_{i}\left(\sqrt{h} h^{i j} G_{A B} \partial_{j} X^{B}\right)=\frac{1}{2} \partial_{A} G_{B C} \partial_{i} X^{B} \partial_{j} X^{C} h^{i j} \sqrt{h},
\end{aligned}
$$

$\left(\partial_{A} \equiv \partial / \partial X^{A}\right)$. If now we assume that the coordinates $\boldsymbol{X} \equiv \boldsymbol{X}(\sigma)$ depend on a single potential function $\sigma\left(x^{i}\right)$, we have the freedom to choose the potential $\sigma$ to be harmonic $\left(\nabla_{h}^{2} \sigma=0\right)$ [10], reducing thus (2.5), (2.6) to

$$
\begin{aligned}
& R_{i j}(h)=\frac{\mathrm{d} l^{2}}{\mathrm{~d} \sigma^{2}} \partial_{i} \sigma \partial_{j} \sigma, \\
& \frac{\mathrm{d}}{\mathrm{d} \sigma}\left(G_{A B} \frac{\mathrm{d} X^{B}}{\mathrm{~d} \sigma}\right)=\frac{1}{2} \partial_{A} G_{B C} \frac{\mathrm{d} X^{B}}{\mathrm{~d} \sigma} \frac{\mathrm{d} X^{C}}{\mathrm{~d} \sigma} .
\end{aligned}
$$

Eqs. (2.8) are the geodesic equations for the target space (2.4). Null geodesics

$$
G_{A B} \dot{X}^{A} \dot{X}^{B}=0 \quad\left(\mathrm{~d} l^{2}=0\right)
$$

(with ${ }^{\cdot} \equiv \mathrm{d} / \mathrm{d} \sigma$ ) lead to a Ricci-flat, hence flat, reduced 3-space of metric $h_{i j}$ [13,14]. In that case, the Laplacian $\nabla_{h}^{2}$ becomes a linear operator, so that an arbitrary number of harmonic functions may be superposed, leading to a multicenter solution

$$
\sigma(\vec{r})=\sum_{i} \frac{c_{i}}{\left|\vec{r}-\vec{r}_{i}\right|}
$$

(up to an additive constant).

The null geodesic condition (2.9) can be interpreted as the condition for the balance between attractive and repulsive forces acting on the "particles" located at the centers $\vec{r}=\vec{r}_{i}$. Defining the mass $M$, electric charge $Q$ and dilatonic charge $D$ of an individual particle from the asymptotic behavior (in the gauge $\phi(\infty)=0$ )

$$
f \sim 1-\frac{2 M}{r}, \quad v \sim \sqrt{2} \frac{Q}{r}, \quad \phi \sim \frac{D}{r}, \quad(r \rightarrow \infty),
$$


with $\sigma=c / r$, we see that the null geodesic condition (2.9) for the metric (2.4) translates into the condition

$$
M^{2}-\eta_{2} Q^{2}+\eta_{1} D^{2}=0
$$

In the case of EMD (which generalizes the Einstein-Maxwell case), the repulsive electrostatic force balances the attractive gravitational and dilatonic forces. However balance can also be achieved in the phantom cases of $\mathrm{EM} \overline{\mathrm{D}}$ (repulsive electrostatic and dilatonic forces) or $\mathrm{E} \overline{\mathrm{MD}}$ (repulsive dilatonic force, the electrostatic force being attractive), but not of EM $\bar{M}$ (all the forces are attractive).

The geodesics for the metric (2.4) are obtained by solving the coupled system of equations

$$
\begin{aligned}
f \ddot{f}-\dot{f}^{2} & =\eta_{2} f \mathrm{e}^{2 \lambda \phi} \dot{v}^{2}, \\
\left(f^{-1} \mathrm{e}^{2 \lambda \phi} \dot{v}\right)^{\cdot} & =0, \\
2 f \ddot{\phi} & =-\eta_{1} \eta_{2} \lambda \mathrm{e}^{2 \lambda \phi} \dot{v}^{2} .
\end{aligned}
$$

To separate this system, we introduce the auxiliary function

$$
\omega=\frac{1}{2} \ln f-\lambda \phi
$$

Equation (2.14) may be integrated to

$$
\dot{v}=\sqrt{2} q \mathrm{e}^{2 \omega},
$$

with $q$ an integration constant. Replacing this in the two other geodesic equations leads to the decoupled system

$$
\begin{aligned}
\ddot{\phi} & =-\eta_{1} \eta_{2} \lambda q^{2} \mathrm{e}^{2 \omega}, \\
\ddot{\omega} & =\eta_{2} \lambda_{+} q^{2} \mathrm{e}^{2 \omega},
\end{aligned}
$$

with

$$
\lambda_{+} \equiv 1+\eta_{1} \lambda^{2}
$$

An obvious first integral of this system is

$$
\lambda \dot{\omega}+\eta_{1} \lambda_{+} \dot{\phi}=k
$$

with $k$ a second integration constant. Eq. (2.19) may also be first integrated to

$$
\dot{\omega}^{2}=\eta_{2} \lambda_{+} q^{2} \mathrm{e}^{2 \omega}+a^{2}
$$

with $a^{2}$ a third integration constant. Finally, the null geodesic equation gives

$$
(\dot{\omega}+\lambda \dot{\phi})^{2}-\eta_{2} q^{2} \mathrm{e}^{2 \omega}+\eta_{1} \dot{\phi}^{2}=0 .
$$

In the generic case $\lambda_{+} \neq 0$, inserting (2.21) and (2.22) into (2.23) leads to the constraint between the integration constants

$$
a^{2}+\eta_{1} k^{2}=0
$$

In the special case $\lambda_{+}=0$, meaning $\eta_{1}=-1$ and $\lambda^{2}=1$, the comparison of (2.21) and (2.22) shows that the constraint (2.24) must again be satisfied.

To analyze the null geodesic solutions, we shall make use of the results of [8]. The static, spherically symmetric metric ansatz used in [8] is

$$
\mathrm{d} s^{2}=\mathrm{e}^{2(\omega+\lambda \phi)} \mathrm{d} t^{2}-\mathrm{e}^{-2(\omega+\lambda \phi)}\left(\mathrm{e}^{4 J} \mathrm{~d} u^{2}+\mathrm{e}^{2 J} \mathrm{~d} \Omega^{2}\right) .
$$


The reduced spatial metric in (2.25) is Euclidean, $\mathrm{d} s_{3}^{2}=\mathrm{d} r^{2}+r^{2} \mathrm{~d} \Omega^{2}$, if $u$ is harmonic, $u=r^{-1}$, and if $J=$ $-\ln |u|$, corresponding to $b=0$ in Eq. (2.18) of [8]. Our master equation (2.22) thus coincides with the master equation (2.14) of [8] with the variable $u \equiv \sigma$, while our constraint (2.24) coincides with the constraint (2.22) of [8] with $b=0$. We shall only discuss here the various solutions which lead to regular (multi)-black holes. Generically, the various centers $\vec{r}=\vec{r}_{i}$ of (2.10) will correspond to horizons $\sigma \rightarrow \pm \infty$ (however there may be other horizons, see below), spacelike infinity $r \rightarrow \infty$ corresponding to $\sigma=0$. We also expect that the solutions will be generically singular unless all the $c_{i}$ in (2.10) are of the same sign, which we will assume positive, thus restricting the domain of $\sigma$ to $\sigma \geq 0$ (this is opposite to the sign convention used in [8]). The solutions can be classified according to the range of $a^{2}$ and the sign of $\bar{q}=\eta_{2} \lambda_{+} q^{2}$.

1. $a^{2}>0, \bar{q}<0$.

$$
\mathrm{e}^{-2 \omega}=\frac{|\bar{q}|}{a^{2}} \cosh ^{2}\left[a\left(\sigma_{0}-\sigma\right)\right]
$$

with $\sigma_{0}$ an integration constant. The metric function $f$ is related to this by the relation (valid whenever $\left.\lambda_{+} \neq 0\right)$

$$
f=c\left(\mathrm{e}^{-2 \omega} \mathrm{e}^{-2 \eta_{1} \lambda k \sigma}\right)^{-1 / \lambda_{+}} .
$$

Here $a$ is real, so $\eta_{1}=-1$ and $k= \pm|a|$ from (2.24). We find that near a horizon,

$$
f \propto \exp [-2|a| \sigma /(1 \mp \lambda)]
$$

which is a nonanalytic function of $r\left(\sigma=r^{-1}\right)$. So we discard this singular solution.

2. $a^{2}>0, \bar{q}=0$.

$$
\omega=a\left(\sigma_{0}-\sigma\right)
$$

This can be divided into two subcases. For $\lambda_{+} \neq 0\left(q^{2}=0\right), f$ is given exactly by $(2.28)$ (with $|a|$ replaced by $a$ ), leading again to a singular horizon. For $\lambda_{+}=0$, we find

$$
\ln f=\frac{\eta_{2} q^{2}}{2 a^{2}} \mathrm{e}^{2 a\left(\sigma_{0}-\sigma\right)}-a \sigma+\text { const. }
$$

also leading to a singular horizon.

3. $a^{2}>0, \bar{q}>0$.

$$
\mathrm{e}^{-2 \omega}=\frac{\bar{q}}{a^{2}} \sinh ^{2}\left[a\left(\sigma_{0}-\sigma\right)\right]
$$

Now, besides the singular horizon at $\sigma \rightarrow \pm \infty$, there is also (if $\sigma_{0}>0$ ) a horizon at $\sigma=\sigma_{0}$ if $\lambda_{+}<0$. The metric can be extended à la Kruskal across this horizon if the metric function (2.27) is an analytic function of $\sigma_{0}-\sigma$, which is the case only if $\lambda_{+}=-2 / p$ ( $p$ positive integer). The values of the coupling constants allowing such regular multicenter solutions are thus

$$
\lambda^{2}=\frac{p+2}{p}, \quad \eta_{1}=-1, \quad \eta_{2}=-1
$$

corresponding to $\overline{\mathrm{MD}}$. The explicit solution is

$$
\begin{aligned}
\mathrm{d} s^{2} & =\left[\frac{\sinh \left[a\left(\sigma_{0}-\sigma\right)\right] \mathrm{e}^{ \pm|\lambda| a \sigma}}{\sinh a \sigma_{0}}\right]^{p} \mathrm{~d} t^{2}-\left[\frac{\sinh \left[a\left(\sigma_{0}-\sigma\right)\right] \mathrm{e}^{ \pm|\lambda| a \sigma}}{\sinh a \sigma_{0}}\right]^{-p} \mathrm{~d} \vec{x}^{2}, \\
A_{0} & =\varepsilon \sqrt{\frac{p}{2}} \frac{\sinh a \sigma}{\sinh \left[a\left(\sigma_{0}-\sigma\right)\right]}, \quad \phi=\frac{1}{2 \lambda}\left((p+2) \ln \left[\frac{\sinh \left[a\left(\sigma_{0}-\sigma\right)\right]}{\sinh a \sigma_{0}}\right] \pm p|\lambda| a \sigma\right),
\end{aligned}
$$


where $\varepsilon= \pm 1$, and we have normalized $\sigma$ by

$$
\sum_{i} c_{i}=1
$$

The total mass, electric charge and dilatonic charge

$$
M=\frac{p a}{2}\left(\operatorname{coth} a \sigma_{0} \mp|\lambda|\right), \quad Q=\varepsilon \sqrt{\frac{p}{2}} \frac{a}{\sinh a \sigma_{0}}, \quad D=-\frac{p a}{2} \operatorname{sign}(\lambda)\left(|\lambda| \operatorname{coth} a \sigma_{0} \mp 1\right)
$$

are related by the balance condition

$$
M^{2}+Q^{2}=D^{2}
$$

Note that in this case the singular centers $\vec{r}=\vec{r}_{i}(\sigma \rightarrow+\infty)$ are hidden behind the horizon (of order $p$ ) $\sigma=\sigma_{0}$, and that the number of connected components of this horizon may be smaller than the number of the centers. As discussed in Subsection IV.C, case (3.b) of [8], where the Penrose diagrams for the onecenter case are given, for the up sign in (2.33) the central singularities are spacelike for $p$ odd or timelike for $p$ even, while for the down sign in (2.33) they are null. The area of each horizon component is infinite, so that these multicenter black holes have a vanishing temperature, i.e. are cold black holes [6, 7].

4. $a^{2}=0, \bar{q}>0$.

$$
\mathrm{e}^{-2 \omega}=\bar{q}\left(\sigma_{0}-\sigma\right)^{2}
$$

leading to

$$
f \propto\left|\sigma_{0}-\sigma\right|^{-2 / \lambda_{+}} .
$$

We must distinguish between three subcases according to the value of $\sigma_{0}$ :

$\alpha) \sigma_{0}>0$. As in the preceding case, $\sigma=\sigma_{0}$ is a regular horizon of order $p$ if $\lambda_{+}=-2 / p$ (implying $\eta_{1}<0$ and $\eta_{2}<0$ so that this case corresponds again to EMD), i.e. for the values of the coupling constants given in (2.32). The explicit solution is

$$
\begin{aligned}
\mathrm{d} s^{2} & =\left(1-\frac{2 M}{p} \sigma\right)^{p} \mathrm{~d} t^{2}-\left(1-\frac{2 M}{p} \sigma\right)^{-p} \mathrm{~d} \vec{x}^{2} \\
A_{0} & =\varepsilon \sqrt{\frac{2}{p}} \frac{M \sigma}{1-2 M \sigma / p}, \quad \phi=\frac{p+2}{2 \lambda} \ln \left(1-\frac{2 M}{p} \sigma\right),
\end{aligned}
$$

with $\sigma$ again normalized by (2.34), and $\sigma_{0}=p /(2 M)$. The total mass $M$, electric charge $Q=\varepsilon(2 / p)^{1 / 2} M$ and dilatonic charge $D=-\lambda M$ are again related by the balance condition (2.36), and the horizon area is again infinite. The global structure in the one-center case is discussed in Subsection IV.D, case (2) of [8]. $\beta) \sigma_{0}<0$. Then the various centers $\vec{r}=\vec{r}_{i}$ each correspond to a connected component of the event horizon $\sigma \rightarrow \infty$, which is regular of order $p$ if $\lambda_{+}=2 / p$, implying $\eta_{2}>0$. This can occur either for $\eta_{1}=+1$, i.e. for normal EMD (the corresponding multicenter solutions have been discussed in [16, 17]), or for $\eta_{1}=-1(\mathrm{EM} \overline{\mathrm{D}})$, i.e. if

$$
\lambda^{2}=\frac{p-2}{p}, \quad \eta_{1}=-1, \quad \eta_{2}=+1,
$$

with $p \geq 2$. The explicit solution is

$$
\begin{aligned}
\mathrm{d} s^{2} & =\left(1+\frac{2 M}{p} \sigma\right)^{-p} \mathrm{~d} t^{2}-\left(1+\frac{2 M}{p} \sigma\right)^{p} \mathrm{~d} \vec{x}^{2}, \\
A_{0} & =\varepsilon \sqrt{\frac{2}{p}} \frac{M \sigma}{1+2 M \sigma / p}, \quad \phi=-\frac{p-2}{2 \lambda} \ln \left(1+\frac{2 M}{p} \sigma\right) .
\end{aligned}
$$


with $\sigma$ normalized by (2.34), and $\sigma_{0}=-p /(2 M)$. The total electric charge and dilatonic charge are again $Q=\varepsilon(2 / p)^{1 / 2} M$ and $D=-\lambda M$, but the balance condition now reads

$$
M^{2}=Q^{2}+D^{2}
$$

For $p=2(\lambda=0)$, we recover the Majumdar-Papapetrou multi-black holes of Einstein-Maxwell theory. For $p>2$, the horizon area is again infinite. The global structure in the one-center case is discussed in Subsection IV.D, case (1) of [8].

$\gamma) \sigma_{0}=0$. Again the horizon $\sigma=\sigma_{0}$ is regular for the values of the coupling constants (2.40), however $f \propto \sigma^{-2 / \lambda_{+}}$does not go to a constant at spacelike infinity $(\sigma \rightarrow 0)$, so that the metric is nonasymptotically flat (NAF). While regular NAF black hole solutions of EMD are known to exist in the normal case [17], it is easy to show that in the present case the Ricci scalar diverges at spatial infinity, so that these solutions do not correspond to regular black holes 1 .

5. $a^{2}<0, \bar{q}>0$. In this case, $\eta_{1}>0$ from (2.24), so that $\lambda_{+}$is positive definite, and $\bar{q}>0$ implies $\eta_{2}>0$, corresponding to normal (non-phantom) EMD [16, 17].

\section{Stationary $\mathbf{E} \bar{M}$ theory}

Now we generalize our investigations to the case of stationary phantom EMD, i.e. we drop the restrictive assumption of staticity. As shown in [15], the target spaces for stationary normal EMD reduced to three dimensions are symmetric spaces in only two cases: $\lambda=0$, and $\lambda=-\sqrt{3}$. The third case $\lambda=-1$ also leads to a symmetric space after enlarging the theory to EMDA [18]. In the following we shall consider the corresponding phantom cases, which lead to symmetric spaces only for $\eta_{1}=+1$ (which will allow us to simplify the notation and write $\eta$ instead of $\eta_{2}$ ). We first discuss the case $\lambda=0$.

Let us first briefly recall the standard procedure [10] for reducing the four-dimensional Einstein-Maxwell theory deriving from the action

$$
S=-\int \mathrm{d}^{4} x \sqrt{-g}\left[\mathscr{R}+F_{\mu v} F^{\mu v}\right]
$$

to an effective three-dimensional gravitating sigma-model theory. Assuming the existence of a timelike Killing vector $\partial_{t}$, the metric may be parametrized by

$$
\mathrm{d} s^{2}=f\left(\mathrm{~d} t-\omega_{i} \mathrm{~d} x^{i}\right)^{2}-f^{-1} h_{i j} \mathrm{~d} x^{i} \mathrm{~d} x^{j},
$$

where $f, \omega_{i}$, and the reduced spatial metric $h_{i j}$ depend only on the three space coordinates $x^{i}(i=1,2,3)$. The stationary Maxwell tensor field may be split into electric and magnetic components deriving from two threedimensional scalar potentials $v$ (electric) and $u$ (magnetic) according to

$$
F_{i 0}=\partial_{i} v, \quad F^{i j}=f h^{-1 / 2} \varepsilon^{i j k} \partial_{k} u
$$

$\left(\varepsilon^{i j k}\right.$ is the totally antisymmetric symbol, with $\varepsilon^{123}=1$ ). Finally, solving the mixed $G_{0}^{i}=0$ Einstein equations enables to trade the three-dimensional vector $\omega_{i}$ for the three-dimensional scalar twist potential $\chi$ such that

$$
\partial_{i} \chi=-f^{2} h^{-1 / 2} h_{i j} \varepsilon^{j k l} \partial_{k} \omega_{l}+2\left(u \partial_{i} v-v \partial_{i} u\right) .
$$

\footnotetext{
${ }^{1}$ This point was overlooked in [8].
} 
The remaining Einstein-Maxwell equations then reduce to the following three-dimensional Ernst equations [19]

$$
\begin{aligned}
f \nabla^{2} \mathscr{E} & =\nabla \mathscr{E} \cdot(\nabla \mathscr{E}+2 \bar{\psi} \nabla \psi), \\
f \nabla^{2} \psi & =\nabla \psi \cdot(\nabla \mathscr{E}+2 \bar{\psi} \nabla \psi), \\
f^{2} R_{i j}(h) & \left.=\operatorname{Re}\left[\frac{1}{2} \mathscr{E}_{,_{i}} \overline{\mathscr{E}}_{\left.{ }_{j}\right)}+2 \psi \mathscr{E},,_{i} \bar{\psi},_{j)}-2 \mathscr{E} \psi,_{i} \bar{\psi},{ }_{j}\right)\right]
\end{aligned}
$$

where the complex Ernst potentials are defined by

$$
\mathscr{E}=f+\mathrm{i} \chi-\bar{\psi} \psi, \quad \psi=v+\mathrm{i} u
$$

and the dot product and covariant derivative $\nabla$ are defined in terms of the spatial metric $h_{i j}$. The gravitating sigma-model equations (3.5) are invariant under an eight-parameter group $S U(2,1)$ of transformations acting on the target space coordinates $\mathscr{E}$ and $\psi$ and leaving invariant the spatial metric $h_{i j}$. The action of this group may be linearized by introducing the complex Kinnersley potentials [20] $(U, V, W)$, related to the Ernst potentials by

$$
\mathscr{E}=\frac{U-W}{U+W}, \quad \psi=\frac{V}{U+W} .
$$

This parametrization is only apparently redundant, the Kinnersley potentials being defined by (3.7) up to a complex local rescaling $\Phi(\vec{x}) \rightarrow \xi(\vec{x}) \Phi(\vec{x})(\xi(\vec{x}) \in C)$ of the "spinor" $\Phi=(U, V, W)$, allowing e.g. to fix the value of the norm $\|\Phi\|^{2}=|U|^{2}+|V|^{2}-|W|^{2}$. The gravistatic potential $f$ is simply related to the Kinnersley potentials by

$$
f=\operatorname{Re} \mathscr{E}+\bar{\psi} \psi=\frac{|U|^{2}+|V|^{2}-|W|^{2}}{|U+W|^{2}} .
$$

Now, consider the general action

$$
S=-\int \mathrm{d}^{4} x \sqrt{-g}\left[\mathscr{R}+\eta F_{\mu v} F^{\mu v}\right]
$$

which describes the Einstein-Maxwell theory if $\eta=+1$, and the Einstein-anti-Maxwell theory if $\eta=-1$. The reduction of the stationary Einstein-anti-Maxwell theory to an effective three-dimensional gravitating sigma model closely parallels that of the Einstein-Maxwell theory, so that it is sufficient to identify the equations where the sign of $\eta$ (or, in other words, the sign of the gravitational constant) comes in. The definition (3.4) of the twist potential becomes

$$
\partial_{i} \chi=-f^{2} h^{-1 / 2} h_{i j} \varepsilon^{j k l} \partial_{k} \omega_{l}+2 \eta\left(u \partial_{i} v-v \partial_{i} u\right),
$$

and the definition of the generalized Ernst potentials

$$
\mathscr{E}=f+\mathrm{i} \chi-\eta \bar{\psi} \psi, \quad \psi=v+\mathrm{i} u,
$$

leads to the generalized Ernst equations

$$
\begin{aligned}
f \nabla^{2} \mathscr{E} & =\nabla \mathscr{E} \cdot(\nabla \mathscr{E}+2 \eta \bar{\psi} \nabla \psi) \\
f \nabla^{2} \psi & =\nabla \psi \cdot(\nabla \mathscr{E}+2 \eta \bar{\psi} \nabla \psi) \\
f^{2} R_{i j}(h) & \left.\left.=\operatorname{Re}\left[\frac{1}{2} \mathscr{E},,_{i} \overline{\mathscr{E}}_{j},+2 \eta \psi \mathscr{E},{ }_{i} \bar{\psi},{ }_{j}\right)-2 \eta \mathscr{E} \psi,{ }_{i} \bar{\psi},{ }_{j}\right)\right]
\end{aligned}
$$

These equations may also be obtained from the equations (3.5) by replacing $\mathscr{E}$ with $\eta \mathscr{E}$ and $f$ with $\eta f$, suggesting the definition of the generalized Kinnersley potentials $(U, V, W)$

$$
\mathscr{E}=\eta \frac{U-W}{U+W}, \quad \psi=\frac{V}{U+W},
$$


in terms of which we express the scalar function $f$ as

$$
f=\eta \frac{|U|^{2}+|V|^{2}-|W|^{2}}{|U+W|^{2}} .
$$

Using (3.13) and (3.14) in equations (3.12), the latter, once written in terms of $(U, V, W)$, take the same form as equations (3.5) do when they are expressed in terms of the previously defined Kinnersley potentials in (3.7). Thus, equations (3.12) remain invariant when acted upon by $S U(2,1)$, which keeps the norm $|U|^{2}+|V|^{2}-|W|^{2}$ invariant so that the sign of $f$ is also invariant. Then, using the third line in (3.12) one concludes that the spatial metric $h_{i j}$ is also invariant.

Asymptotically flat field configurations are such that, up to a gauge transformation, $f(\infty)=1$ with the three other scalar potentials $\chi, v$ and $u$ vanishing at spatial infinity, leading, from the third equation (3.12), to an asymptotically flat reduced spatial metric $h_{i j}$. Thus, at spatial infinity $(|\vec{x}| \rightarrow \infty)$, the spinor $\Phi_{\eta}(\infty)$ representing any asymptotically flat solution is of the form

$$
\begin{array}{ll}
\Phi_{+}(\infty)=(1,0,0) & (\eta=+1), \\
\Phi_{-}(\infty)=(0,0,1) & (\eta=-1) .
\end{array}
$$

The asymptotic behavior (3.15) is preserved, up to a complex rescaling, by the transformations of the subgroup $U(1,1)$ mixing $V$ and $W$ on the one hand, by the transformations of the subgroup $U(1)$ changing the phase of $U$ on the other hand, and more generally by the product of two such transformations up to a complex rescaling, i.e. by the transformations of the isotropy subgroup $H_{+}=S[U(1,1) \times U(1)] \subset S U(2,1)$. The coset space for stationary Einstein-Maxwell theory $(\eta=+1)$ is thus $S U(2,1) / S[U(1,1) \times U(1)]$ [9]. Similarly, the asymptotic behavior (3.16) is preserved (up to a rescaling) by the $U(2)$ transformations mixing $U$ and $V$ on the one hand, and by the $U(1)$ transformations acting on $W$ on the other hand, generating the isotropy subgroup $H_{-}=S[U(2) \times U(1)]$. So the coset space for stationary Einstein-anti-Maxwell theory $(\eta=-1)$ is $S U(2,1) / S[U(2) \times U(1)]$.

A simple application is the generation of static phantom charged black holes from the Schwarzschild solution $f_{0}=1-2 M_{0} / r$. The spinor representing the Schwarzschild solution is (up to a rescaling)

$$
\Phi_{0}=\left(M_{0}, 0, r-M_{0}\right)
$$

Acting on this by $S O(2)$ transformations parametrized by an angle $\alpha$ leads to

$$
\Phi=\left(M_{0} \cos \alpha, M_{0} \sin \alpha, r-M_{0}\right)
$$

which corresponds to the phantom Reissner-Nordström solution [5]

$$
\mathrm{d} s^{2}=\left(1-\frac{r_{+}}{\bar{r}}\right)\left(1-\frac{r_{-}}{\bar{r}}\right) \mathrm{d} t^{2}-\left(1-\frac{r_{+}}{\bar{r}}\right)^{-1}\left(1-\frac{r_{-}}{\bar{r}}\right)^{-1} \mathrm{~d} \bar{r}^{2}-\bar{r}^{2} \mathrm{~d} \Omega^{2}, \quad \psi=\frac{Q}{\bar{r}},
$$

with $\bar{r}=r+r_{-}, r_{+}=M_{0}(1+\cos \alpha), r_{-\overline{2}}=-M_{0}(1-\cos \alpha), Q=M_{0} \sin \alpha$. The black hole mass $M=\left(r_{+}+\right.$ $\left.r_{-}\right) / 2=M_{0} \cos \alpha$ vanishes for $\alpha=\pi / 2$ 2. Then the black hole mass becomes negative, until the limiting value $M=-M_{0}$ is attained, corresponding (not surprisingly) to the singular Schwarzschild solution with negative mass.

\footnotetext{
${ }^{2}$ The corresponding $S O(2)$ transformation is $U=-V_{0}, V=U_{0}$. In the EM case, this transformation generates from the Schwarzschild solution the non-asymptotically flat Bertotti-Robinson solution [21], which is not possible here.
} 


\section{Stationary phantom Kaluza-Klein theory}

Let us consider the vacuum Einstein-Hilbert action in 5D

$$
S_{5}=-\int \mathrm{d}^{5} x{\sqrt{-{ }^{(5)} g}}^{(5)} \mathscr{R} .
$$

The theory may be reduced à la Kaluza-Klein to four dimensions by assuming the existence of a Killing vector $\partial_{5}$ which may be either spacelike (as usually assumed), corresponding to $\eta=+1$, or timelike (this is the phantom case), corresponding to $\eta=-1$. The generalized Kaluza-Klein ansatz

$$
\mathrm{d} s_{(5)}^{2}=\mathrm{e}^{2 \phi / \sqrt{3}(4)} g_{\mu v}\left(x^{\alpha}\right) \mathrm{d} x^{\mu} \mathrm{d} x^{\nu}-\eta \mathrm{e}^{-4 \phi / \sqrt{3}}\left(\mathrm{~d} x^{5}+2 A_{\mu} \mathrm{d} x^{\mu}\right)^{2},
$$

leads to the reduced action

$$
S_{4}=-\int \mathrm{d}^{4} x \sqrt{-{ }^{(4)} g}\left[{ }^{(4)} \mathscr{R}-2^{(4)} g^{\mu v} \partial_{\mu} \phi \partial_{\nu} \phi+\eta \mathrm{e}^{-2 \sqrt{3} \phi} F_{\mu v} F^{\mu v}\right]
$$

for normal $(\eta=+1)$, or phantom $(\eta=-1)$ Einstein-Maxwell-dilaton (EMD or E $\bar{M} D)$ theory [8] with dilaton coupling constant $\lambda=-\sqrt{3}$.

The four-dimensional theory (4.3) may further be reduced to three dimensions by assuming stationarity, i.e. the existence of a second, timelike, Killing vector $\partial_{0}$. Equivalently, we can directly reduce the five-dimensional theory (4.1) to three dimensions by assuming the existence of two commuting Killing vectors $\partial_{0}$ and $\partial_{5}$, with the first timelike and the second spacelike or timelike according to the sign of $\eta$. We require the reduced threespace to be topologically Euclidean, so that the signature of the five-dimensional spacetime metric is +---for $\eta=+1$, and ++--- for $\eta=-13$. The five-to-three reduction is parametrized according to the metric ansatz [22]

$$
d s_{(5)}^{2}=\lambda_{a b}\left[\mathrm{~d} x^{a}+V^{a}{ }_{i} \mathrm{~d} x^{i}\right]\left[\mathrm{d} x^{b}+V^{b}{ }_{j} \mathrm{~d} x^{j}\right]-\tau^{-1} h_{i j} \mathrm{~d} x^{i} \mathrm{~d} x^{j},
$$

where $a, b=0,5$ and $\tau=\left|\operatorname{det}\left[\lambda_{a b}\right]\right|$. The 3 -vectors $V^{a}{ }_{i}$ may be dualized to scalar twist potentials $\omega_{a}$ via the relations

$$
\omega_{a, i}=|h|^{-1 / 2} \tau h_{i l} \lambda_{a b} \varepsilon^{j k l} V^{b}{ }_{j, k} .
$$

The remaining field equations derived from (4.1) may be written as the three-dimensional sigma-model system [22]

$$
\begin{aligned}
& R_{i j}(h)=\frac{1}{4} \operatorname{tr}\left[\chi^{-1} \partial_{i} \chi \chi^{-1} \partial_{j} \chi\right] \\
& \nabla_{i}\left(\chi^{-1} \partial^{i} \chi\right)=0
\end{aligned}
$$

where $\chi$ is the $3 \times 3$ symmetric and unimodular Maison matrix defined (in block form) by

$$
\chi=\left[\begin{array}{cc}
\lambda+\tau^{-1} \omega \omega^{T} & \tau^{-1} \omega \\
\tau^{-1} \omega^{T} & \tau^{-1}
\end{array}\right]
$$

The field equations (4.6), (4.7) are invariant under an $\operatorname{SL}(3, \mathbb{R})$ group of transformations $P$ acting bilinearly on the matrix $\chi$ :

$$
\chi^{\prime}=P^{T} \chi P
$$

\footnotetext{
${ }^{3}$ The phantom $(\eta=-1)$ Kaluza-Klein theory is a two-time theory. For this reason, we do not require the fifth dimension to be compact, which would lead unavoidably to closed timelike curves.
} 
For asymptotically flat configurations $(\lambda(\infty)=\operatorname{diag}(1,-\eta), \omega(\infty)=0)$, the matrix $\chi$ is asymptotic to

$$
\chi(\infty)=\left[\begin{array}{ccc}
1 & 0 & 0 \\
0 & -\eta & 0 \\
0 & 0 & 1
\end{array}\right]
$$

It follows that the isotropy group at spatial infinity is $H_{+}=S O(2,1)$ for $\eta=+1$, and $H_{-}=S O(3)$ for $\eta=-1$. Thus, the coset space for phantom Kaluza-Klein theory is $S L(3, \mathbb{R}) / S O(3)$ (instead of $S L(3, \mathbb{R}) / S O(2,1)$ in the normal case).

To illustrate the applications of this sigma model, we shall generate from the four-dimensional Kerr metric embedded in five dimensions (with a timelike fifth dimension),

$$
\mathrm{d} s_{(5)}^{2}=\frac{g_{0}}{\Sigma_{0}}\left(\mathrm{~d} t-\Omega_{0} \mathrm{~d} \varphi\right)^{2}+\left(\mathrm{d} x^{5}\right)^{2}-\frac{\Sigma_{0}}{g_{0}}\left[\frac{g_{0}}{\Delta_{0}} \mathrm{~d} r^{2}+g_{0} \mathrm{~d} \theta^{2}+\Delta_{0} \sin ^{2} \theta \mathrm{d} \varphi^{2}\right],
$$

where

$$
\begin{array}{cc}
\Delta_{0}=r^{2}-2 M_{0} r+a_{0}^{2}, & \Sigma_{0}=r^{2}+a_{0}^{2} \cos ^{2} \theta, \\
g_{0}=\Sigma_{0}-2 M_{o} r, & \Omega_{0}=-2 a_{0} M_{0} \frac{r \sin ^{2} \theta}{g_{0}},
\end{array}
$$

a charged rotating black hole solution of the phantom theory E $\overline{M D}$. The metric (4.11) is of the form (4.4) with $V_{3}^{0}=-\Omega_{0}$. Using the duality equation (4.5), we obtain

$$
\omega_{0}=2 a_{0} M_{0} \frac{\cos \theta}{\Sigma_{0}}
$$

leading to the seed Maison matrix

$$
\chi_{0}=g_{0}^{-1}\left[\begin{array}{ccc}
\left(r-2 M_{0}\right)^{2}+a_{0}^{2} \cos ^{2} \theta & 0 & 2 a_{0} M_{0} \cos \theta \\
0 & g_{0} & 0 \\
2 a_{0} M_{0} \cos \theta & 0 & \Sigma_{0}
\end{array}\right] .
$$

Applying to this seed the $S O(3)$ group transformation

$$
\chi=P^{T} \chi_{0} P, \quad P=\left[\begin{array}{ccc}
\cos \alpha & -\sin \alpha & 0 \\
\sin \alpha & \cos \alpha & 0 \\
0 & 0 & 1
\end{array}\right]
$$

we obtain the Maison matrix for the charged solution

$$
\chi(r)=\frac{1}{g_{0}}\left[\begin{array}{ccc}
g_{0}-2 M_{0} \cos ^{2} \alpha\left(r-2 M_{0}\right) & 2 M_{0} \sin \alpha \cos \alpha\left(r-2 M_{0}\right) & 2 a_{0} M_{0} \cos \alpha \cos \theta \\
2 M_{0} \sin \alpha \cos \alpha\left(r-2 M_{0}\right) & g_{0}-2 M_{0} \sin ^{2} \alpha\left(r-2 M_{0}\right) & -2 a_{0} M_{0} \sin \alpha \cos \theta \\
2 a_{0} M_{0} \cos \alpha \cos \theta & -2 a_{0} M_{0} \sin \alpha \cos \theta & \Sigma_{0}
\end{array}\right] .
$$

After inverse dualization, this leads to the 5-metric

$$
\mathrm{d} s_{(5)}^{2}=\frac{\Sigma}{\Sigma_{0}} \Lambda^{2}+\frac{4 Q}{\Sigma_{0}} r \Lambda \Psi+\frac{g}{\Sigma_{0}} \Psi^{2}-\frac{\Sigma_{0}}{g_{0}}\left[\frac{g_{0}}{\Delta_{0}} \mathrm{~d} r^{2}+g_{0} \mathrm{~d} \theta^{2}+\Delta_{0} \sin ^{2} \theta \mathrm{d} \varphi^{2}\right],
$$

where

$$
\begin{array}{cl}
\Sigma=\Sigma_{0}-4 \sigma r, & g=g_{0}+4 \sigma r \\
\Lambda=\mathrm{d} x^{5}-\frac{2 J Q}{(M-\sigma)} \frac{r \sin ^{2} \theta}{g_{0}} \mathrm{~d} \varphi, & \Psi=\mathrm{d} t+2 J \frac{r \sin ^{2} \theta}{g_{0}} \mathrm{~d} \varphi
\end{array}
$$


and

$$
\begin{aligned}
& M=M_{0}\left(1+\cos ^{2} \alpha\right) / 2, Q=M_{0} \sin \alpha \cos \alpha, \\
& J=a_{0} M_{0} \cos \alpha, \sigma=M_{0} \sin ^{2} \alpha / 2,
\end{aligned}
$$

are the mass, electric charge, angular momentum and rescaled dilatonic charge (the usual dilatonic charge is $D=\sqrt{3} \sigma$ ) defined from the asymptotic behavior of the charged solution

$$
\chi(r \rightarrow+\infty) \sim\left[\begin{array}{ccc}
1-\frac{2(M-\sigma)}{r} & \frac{2 Q}{r} & \frac{2 J \cos \theta}{r^{2}} \\
\frac{2 Q}{r} & 1-\frac{4 \sigma}{r} & 0 \\
\frac{2 J \cos \theta}{r^{2}} & 0 & 1+\frac{2(M+\sigma)}{r}
\end{array}\right] .
$$

Note that only three of these charges are independent, as $Q^{2}=2 \sigma(M-\sigma)$, with $0 \leq \sigma \leq M$. The seed Kerr parameters may be recovered from these by

$$
M_{0}=M+\sigma, \quad a_{0}=\frac{|J|}{\sqrt{M^{2}-\sigma^{2}}} .
$$

The solution (4.18) is the phantom version of Rasheed's rotating solution [23] with vanishing magnetic charge $(P=0)$, from which it may be obtained by making the substitution $Q^{2} \rightarrow-Q^{2}$ and changing the sign of the dilatonic charge.

The Kaluza-Klein reduction (4.2) of (4.18) leads to the four-dimensional solution

$$
\begin{aligned}
\mathrm{d} s_{(4)}^{2} & =\frac{g_{0}}{\sqrt{\Sigma_{0} \Sigma}}\left[\mathrm{d} t+2 J \frac{r \sin ^{2} \theta}{g_{0}} \mathrm{~d} \varphi\right]^{2}-\frac{\sqrt{\Sigma_{0} \Sigma}}{g_{0}}\left[\frac{g_{0}}{\Delta_{0}} \mathrm{~d} r^{2}+g_{0} \mathrm{~d} \theta^{2}+\Delta_{0} \sin ^{2} \theta \mathrm{d} \varphi^{2}\right], \\
A & =Q \frac{r}{\Sigma}\left[\mathrm{d} t-\frac{J}{M-\sigma} \sin ^{2} \theta \mathrm{d} \varphi\right], \quad \mathrm{e}^{2 \phi / \sqrt{3}}=\sqrt{\frac{\Sigma_{0}}{\Sigma}} .
\end{aligned}
$$

which describes an asymptotically flat rotating phantom dilatonic black hole with electric charge $Q$ and magnetic dipole moment

$$
\mu=\frac{J Q}{M-\sigma}=a_{0} M_{0} \sin \alpha
$$

The corresponding gyromagnetic ratio

$$
g=\frac{2 M \mu}{Q J}=2+\tan ^{2} \alpha
$$

is for $\alpha \neq 0$ larger than 2. In the static $\left(a_{0}=0\right)$ case, the solution (4.24)-(4.25) reduces to the "cosh" solution of [8] with $\lambda=-\sqrt{3}$,

$$
\begin{aligned}
\mathrm{d} s_{(4)}^{2} & =\left(1-\frac{r_{+}}{\bar{r}}\right)\left(1-\frac{r_{-}}{\bar{r}}\right)^{-1 / 2} \mathrm{~d} t^{2}-\left(1-\frac{r_{+}}{\bar{r}}\right)^{-1}\left(1-\frac{r_{-}}{\bar{r}}\right)^{1 / 2}\left[\mathrm{~d} \bar{r}^{2}+\left(\bar{r}-r_{+}\right)\left(\bar{r}-r_{-}\right) \mathrm{d} \Omega^{2}\right], \\
A_{0} & =\frac{Q}{\bar{r}}, \quad \mathrm{e}^{4 \phi / \sqrt{3}}=1-\frac{r_{-}}{\bar{r}},
\end{aligned}
$$

where $\bar{r}=r-4 \sigma$, and $r_{+}=2(M-\sigma) \geq 0, r_{-}=-4 \sigma \leq 0$.

The five-dimensional solution (4.18) or its Kaluza-Klein reduction (4.24) represent a black hole with the same horizons

$$
r=r_{h \pm} \equiv M_{0} \pm \sqrt{M_{0}^{2}-a_{0}^{2}}
$$


(if $a_{0}^{2} \leq M_{0}^{2}$ ) and ring singularity $r=0, \cos \theta=0$ as the seed Kerr black hole. The four-dimensional metric (4.24) is also singular on the surfaces $\Sigma(r, \theta)=0$,

$$
r=r_{s \pm}(\theta) \equiv 2 \sigma \pm\left(4 \sigma^{2}-a_{0}^{2} \cos ^{2} \theta\right)^{1 / 2}
$$

The largest value of $r_{s+}(\theta)$ is $4 \sigma$, so this singularity will be naked unless

$$
4 \sigma<M_{0}+\sqrt{M_{0}^{2}-a_{0}^{2}}, \quad \text { or } \quad \sqrt{M_{0}^{2}-a_{0}^{2}}>-M_{0} \cos (2 \alpha) .
$$

This regularity condition is satisfied if either $\cos (2 \alpha)>0$, or if $\cos (2 \alpha)<0$ and $\left|a_{0}\right|<2|Q|$. However at the five-dimensional level (metric (4.18) $), \Sigma=0$ is simply the static limit associated with the time coordinate $x^{5}$, and is a mere coordinate singularity. So, in the five-dimensional setting, the four-dimensional singularity (4.30) is actually a spurious singularity due to the Kaluza-Klein reduction.

When the charging parameter $\alpha$ takes the value $\alpha=\pi / 2$, something curious happens. For this value, the physical charges are

$$
M=\sigma, \quad Q=0, \quad J=0
$$

(implying $\Sigma=g_{0}$ and $g=\Sigma_{0}$ ), so that at first sight the solution is electrically neutral and static. Actually it is neither, as the dipole magnetic moment $\mu$ given by (4.26) goes to the finite value $a_{0} M_{0}$ for $\alpha \rightarrow \pi / 2$, showing that the static $(J=0)$ four-dimensional metric (4.24) is generated by the magnetic dipole field

$$
A_{3}=-a_{0} M_{0} r \sin ^{2} \theta / \Sigma .
$$

Again, this is just an effect of the Kaluza-Klein reduction. The five-dimensional metric (4.18) reads, for $\alpha=$ $\pi / 2$,

$$
\mathrm{d} s_{(5)}^{2}=\mathrm{d} t^{2}+\frac{g_{0}}{\Sigma_{0}}\left(\mathrm{~d} x^{5}+\Omega_{0} \mathrm{~d} \varphi\right)^{2}-\frac{\Sigma_{0}}{g_{0}}\left[\frac{g_{0}}{\Delta_{0}} \mathrm{~d} r^{2}+g_{0} \mathrm{~d} \theta^{2}+\Delta_{0} \sin ^{2} \theta \mathrm{d} \varphi^{2}\right],
$$

which is just the embedded Kerr metric (4.11) with the time coordinates $t=x^{0}$ and $x^{5}$ exchanged and the angular momentum flipped. For the intermediate value $\alpha=\pi / 4$ (corresponding to the maximum electric charge $Q=M-\sigma=M_{0} / 2$ ), we find $\Sigma=g$, so that the reduction of (4.18) can be indifferently carried out relative to $\partial_{0}$ or to $\partial_{5}$, leading to the same reduced four-dimensional fields, provided the sign of $\varphi$ is flipped. For other values of $\alpha$, the exchange $\alpha=\pi / 2-\alpha^{\prime}, \varphi=-\varphi^{\prime}$ is equivalent to the exchange $(\Sigma, g)=\left(g^{\prime}, \Sigma^{\prime}\right)$ between the two time coordinates in (4.18), leading to the exchange between the effective four-dimensional charges

$$
\begin{aligned}
M^{\prime} & =\frac{M+3 \sigma}{2}, & \sigma^{\prime} & =\frac{M-\sigma}{2}, \\
J^{\prime} & =\frac{J Q}{M-\sigma}, & Q^{\prime} & =Q .
\end{aligned}
$$

The reason for this becomes clear if we realize that the $S L(3, \mathbb{R})$ group of transformations (4.9) includes a $G L(2, R)$ subgroup of transformations

$$
P=\left[\begin{array}{cc}
\Pi & 0 \\
0 & -|\Pi|^{-1}
\end{array}\right]
$$

where the $2 \times 2$ matrix $\Pi$ defines a coordinate transformation in the space of the two timelike cyclic coordinates,

$$
\left[\begin{array}{l}
x^{0} \\
x^{5}
\end{array}\right]=\Pi\left[\begin{array}{l}
x^{\prime} \\
x^{\prime} 5
\end{array}\right] .
$$


The charging transformation (4.16) is such a $G L(2, R)$ transformation. This means that the five-dimensional "charged" black hole metric (4.18) is simply the five-dimensional (phantom) Kerr black string metric (4.11) transformed to an unfamiliar coordinate system, while the four-dimensional charged black hole solution (4.24)(4.25) is simply the Kaluza-Klein reduction of the phantom Kerr black string relative to a linear combination of the Killing vectors $\partial_{0}$ and $\partial_{5}$.

\section{Stationary EMDDA theory}

Einstein-Maxwell-dilaton-axion theory is a truncation of the bosonic sector of four-dimensional $\mathrm{N}=4$ supergravity, defined by the action

$$
S=-\int \mathrm{d} x^{4} \sqrt{-g}\left[\mathscr{R}-2 \partial_{\mu} \phi \partial^{\mu} \phi-\frac{1}{2} \mathrm{e}^{4 \phi} \partial_{\mu} \kappa \partial^{\mu} \kappa+\eta \mathrm{e}^{-2 \phi} F_{\mu v} F^{\mu v}+\kappa F_{\mu v} \tilde{F}^{\mu v}\right],
$$

where $\kappa$ is the pseudoscalar axion, and $\tilde{F}^{\mu v}=\frac{1}{2} E^{\mu \nu \lambda \tau} F_{\lambda \tau}$ 4. The sign $\eta=+1$, corresponding to normal EMDA, can be changed to $\eta=-1$, corresponding to phantom EMDA, by the formal translation $\phi \rightarrow \phi+\mathrm{i} \pi / 2$. Reduction of the stationary theory to three dimensions is achieved in a manner similar to that of EinsteinMaxwell theory, with a metric parametrized as in (3.2), and electric $(v)$, magnetic $(u)$, and twist $(\chi)$ potentials defined by

$$
\begin{aligned}
& F_{i 0}=\frac{1}{\sqrt{2}} \partial_{i} v, \quad \kappa \tilde{F}^{i j}+\eta \mathrm{e}^{-2 \phi} F^{i j}=\frac{f}{\sqrt{2}} h^{-1 / 2} \varepsilon^{i j k} \partial_{k} u, \\
& \partial_{i} \chi+v \partial_{i} u-u \partial_{i} v=-f^{2} h^{-1 / 2} h_{i j} \varepsilon^{j k l} \partial_{k} \omega_{l} .
\end{aligned}
$$

As shown in [18] (where we replace whenever necessary $\mathrm{e}^{2 \phi}$ by $\eta \mathrm{e}^{2 \phi}$ ), this reduction leads to the symmetric target space metric

$$
\begin{aligned}
\mathrm{d} l^{2}= & \frac{1}{2} f^{-2} \mathrm{~d} f^{2}+\frac{1}{2} f^{-2}(\mathrm{~d} \chi+v \mathrm{~d} u-u \mathrm{~d} v)^{2}-\eta f^{-1} \mathrm{e}^{-2 \phi} \mathrm{d} v^{2} \\
& -\eta f^{-1} \mathrm{e}^{2 \phi}(\mathrm{d} u-\kappa \mathrm{d} v)^{2}+2 \mathrm{~d} \phi^{2}+\frac{1}{2} \mathrm{e}^{4 \phi} \mathrm{d} \kappa^{2}
\end{aligned}
$$

with signature $(++++--)$ in the normal case $\eta=+1$ and $(++++++)$ in the phantom case $\eta=-1$. This target space is invariant under a ten-parameter group $\operatorname{Sp}(4, \mathbb{R})$ of transformations [18].

The symplectic group $S p(4, \mathbb{R})$ is the group of real $4 \times 4$ matrices $M$ satisfying

$$
M^{T} J M=J, \quad J=\left[\begin{array}{cc}
0 & \sigma_{0} \\
-\sigma_{0} & 0
\end{array}\right],
$$

where $\sigma_{0}$ is the $2 \times 2$ identity matrix. A base of ten traceless $4 \times 4$ matrices generating the algebra $s p(4, \mathbb{R})$ has been constructed in [18]:

$$
\begin{aligned}
& V_{a}=\frac{1}{2}\left[\begin{array}{cc}
0 & \sigma_{a} \\
\sigma_{a} & 0
\end{array}\right], W_{a}=\frac{1}{2}\left[\begin{array}{cc}
\sigma_{a} & 0 \\
0 & -\sigma_{a}
\end{array}\right], \\
& U_{a}=\frac{1}{2}\left[\begin{array}{cc}
0 & \sigma_{a} \\
-\sigma_{a} & 0
\end{array}\right], U_{2}=\frac{1}{2}\left[\begin{array}{cc}
\sigma_{2} & 0 \\
0 & \sigma_{2}
\end{array}\right],
\end{aligned}
$$

( $a=0,1,3, \sigma_{1}=\sigma_{x}, \sigma_{2}=i \sigma_{y}, \sigma_{3}=\sigma_{z}$ with $\sigma_{x}, \sigma_{y}, \sigma_{z}$ the Pauli matrices). The six generators $V_{a}$ and $W_{a}$ are symmetrical, and the four generators $U_{a}$ and $U_{2}$ are antisymmetrical.

\footnotetext{
${ }^{4}$ We use the convention $E^{\mu \nu \lambda \tau} \equiv|g|^{-1 / 2} \varepsilon^{\mu \nu \lambda \tau}$, with $\varepsilon^{0123}=-1$.
} 
As also shown in [18], the reduced field equations derive from the gravitating sigma-model action

$$
S_{3}=\int \mathrm{d}^{3} x \sqrt{h}\left[R(h)+\frac{1}{4} \operatorname{tr}\left(\nabla M \nabla M^{-1}\right)\right],
$$

with the symmetrical symplectic matrix representative

$$
M=\left[\begin{array}{cc}
P^{-1} & P^{-1} Q \\
Q P^{-1} & P+Q P^{-1} Q
\end{array}\right]
$$

where the $2 \times 2$ block matrices $P$ and $Q$ are

$$
P=\mathrm{e}^{-2 \phi}\left[\begin{array}{cc}
f \mathrm{e}^{2 \phi}-\eta v^{2} & -\eta v \\
-\eta v & -\eta
\end{array}\right], Q=\left[\begin{array}{cc}
v w-\chi & w \\
w & -\kappa
\end{array}\right] \quad(w=u-\kappa v)
$$

The matrix representative $M$ parametrizes an element of the coset space $S p(4, \mathbb{R}) / H_{\eta}$, where $H_{\eta}$ is the isotropy subgroup that preserves asymptotic behavior. To determine this subgroup, we consider asymptotically flat solutions for which $f(\infty)=1$ and the five other target space coordinates go to zero. For normal EMDA $(\eta=+1)$, we find from (5.9) and (5.10) that

$$
M_{+}(\infty)=\left[\begin{array}{cc}
\sigma_{3} & 0 \\
0 & \sigma_{3}
\end{array}\right]
$$

This remains invariant under the $S p(4, \mathbb{R})$ transformations

$$
M^{\prime}=P^{T} M P
$$

generated by

$$
\operatorname{lie}\left(H_{+}\right)=\left(V_{1}, W_{1}, U_{0}, U_{3}\right) .
$$

These matrices generate the $u(1,1)$ algebra with center $U_{3}$ : $\left[W_{1}, V_{1}\right]=U_{0},\left[U_{0}, V_{1}\right]=W_{1},\left[W_{1}, U_{0}\right]=V_{1}$ and $\left[V_{1}, U_{3}\right]=\left[W_{1}, U_{3}\right]=\left[U_{0}, U_{3}\right]=0$. It follows that the coset for EMDA is $\operatorname{Sp}(4, \mathbb{R}) / U(1,1) \sim S O(3,2) /(S O(2,1) \times$ $S O(2))$.

For phantom EMDA $(\eta=-1), M_{-}(\infty)$ is the $4 \times 4$ identity matrix,

$$
M_{-}(\infty)=\left[\begin{array}{cc}
\sigma_{0} & 0 \\
0 & \sigma_{0}
\end{array}\right]
$$

This matrix remains invariant under the subgroup generated by the antisymmetrical matrices

$$
\operatorname{lie}\left(H_{-}\right)=\left(U_{a}, U_{2}\right) \text {. }
$$

These matrices generate the $u(2)$ algebra with center $U_{0}:\left[U_{i}, U_{j}\right]=-\varepsilon_{i j k} U_{k},\left[U_{0}, U_{i}\right]=0, i, j, k=1,2,3$ and $\varepsilon_{123}=+1$. It follows that the coset for EM DA is $S p(4, \mathbb{R}) / U(2) \sim S O(3,2) /(S O(3) \times S O(2))$.

A third four-dimensional subgroup of $\operatorname{Sp}(4, \mathbb{R})$ is $G L(2, \mathbb{R})$. The choice of this as isotropy subgroup leads to the coset $S p(4, \mathbb{R}) / G L(2, \mathbb{R}) \sim S O(3,2) /(S O(2,1) \times S O(1,1))$ [24], which is the coset for Euclidean EMDA [25].

Let us now, as in the previous section, apply the EM $\bar{M}$ A sigma model to the generation of a charged rotating black hole solution from the four-dimensional Kerr metric given by (4.11) (without the fifth dimension). The twist potential $\chi_{0}$ is the opposite of $\omega_{0}$ in (4.14), leading to the seed matrix representative

$$
M_{0}=g_{0}^{-1}\left[\begin{array}{cccc}
\Sigma_{0} & 0 & 2 a_{0} M_{0} \cos \theta & 0 \\
0 & g_{0} & 0 & 0 \\
2 a_{0} M_{0} \cos \theta & 0 & \left(r-2 M_{0}\right)^{2}+a_{0}^{2} \cos ^{2} \theta & 0 \\
0 & 0 & 0 & g_{0}
\end{array}\right] \text {. }
$$


Applying to this the $S p(4, \mathbb{R})$ transformation (5.12) with

$$
P=\left[\begin{array}{cccc}
\cos \alpha & -\sin \alpha & 0 & 0 \\
\sin \alpha & \cos \alpha & 0 & 0 \\
0 & 0 & \cos \alpha & -\sin \alpha \\
0 & 0 & \sin \alpha & \cos \alpha
\end{array}\right]
$$

we obtain the matrix representing the charged rotating solution

$$
M=g_{0}^{-1}\left[\begin{array}{cccc}
r(r-2 D)+a_{0}^{2} c^{2} & -\sqrt{2} Q r & 2 a_{0} M c & -a_{0} \sqrt{2} Q c \\
-\sqrt{2} Q r & r(r-2 M)+a_{0}^{2} c^{2} & -a_{0} \sqrt{2} Q c & 2 a_{0} D c \\
2 a_{0} M c & -a_{0} \sqrt{2} Q c & \left(r-2 M_{0}\right)(r-2 M)+a_{0}^{2} c^{2} & 2 M_{0}\left(r-2 M_{0}\right) \\
-a_{0} \sqrt{2} Q c & 2 a_{0} D c & 2 M_{0}\left(r-2 M_{0}\right) & \left(r-2 M_{0}\right)(r-2 D)+a_{0}^{2} c^{2}
\end{array}\right],
$$

with $c \equiv \cos \theta$, and

$$
M=M_{0} \cos ^{2} \alpha, Q=\sqrt{2} M_{0} \sin \alpha \cos \alpha, D=M_{0} \sin ^{2} \alpha \quad\left(M_{0}=M+D, Q^{2}=2 M D\right) .
$$

The corresponding fields, obtained by identifying (5.9) with (5.18) and performing inverse dualization, are

$$
\begin{aligned}
\mathrm{d} s^{2} & =\frac{g_{0}}{\Sigma}\left[\mathrm{d} t+2 J \frac{r \sin ^{2} \theta}{g_{0}} \mathrm{~d} \varphi\right]^{2}-\frac{\Sigma}{g_{0}}\left[\frac{g_{0}}{\Delta_{0}} \mathrm{~d} r^{2}+g_{0} \mathrm{~d} \theta^{2}+\Delta_{0} \sin ^{2} \theta \mathrm{d} \varphi^{2}\right] \\
A & =Q \frac{r}{\Sigma}\left[\mathrm{d} t-a_{0} \sin ^{2} \theta \mathrm{d} \varphi\right], \quad \mathrm{e}^{2 \phi}=\frac{\Sigma_{0}}{\Sigma}, \quad \kappa=-\frac{2 a_{0} D \cos \theta}{\Sigma_{0}},
\end{aligned}
$$

with

$$
\Sigma=r(r-2 D)+a_{0}^{2} \cos ^{2} \theta, \quad J=a_{0} M .
$$

In the static $\left(a_{0}=0\right)$ case, this solution reduces to the cosh solution of [8] with $\lambda=-1$,

$$
\begin{aligned}
\mathrm{d} s^{2} & =\left(1-\frac{r_{+}}{\bar{r}}\right) \mathrm{d} t^{2}-\left(1-\frac{r_{+}}{\bar{r}}\right)^{-1}\left[\mathrm{~d} \bar{r}^{2}+\left(\bar{r}-r_{+}\right)\left(\bar{r}-r_{-}\right) \mathrm{d} \Omega^{2}\right], \\
A_{0} & =\frac{Q}{\bar{r}}, \quad \mathrm{e}^{2 \phi}=1-\frac{r_{-}}{\bar{r}},
\end{aligned}
$$

with $r_{+}=2 M \geq 0, r_{-}=-2 D \leq 0$, and $\bar{r}=r+r_{-}$.

The solution (5.20) of phantom EMDA represents a rotating, electrically charged black hole with mass $M$, electric charge $Q$, dilatonic charge $D$, angular momentum $J$, magnetic dipole charge $a_{0} Q$, and axionic dipole charge $a_{0} D$. It is formally identical to the charged rotating black hole solution of normal EMDA [26], the only difference being that the dilatonic charge $D$ is negative in the case of EMDA $\left(Q^{2}=-2 M D\right)$ and positive in the present case of $E \bar{M} D A\left(Q^{2}=2 M D\right)$. A consequence is that (as in the case of the phantom $\lambda=-\sqrt{3}$ black hole (4.24) ) the outermost singular surface $(\Sigma(r, \theta)=0)$,

$$
r_{s+}(\theta)=D+\sqrt{D^{2}-a_{0}^{2} \cos ^{2} \theta}
$$

may lie (in part) outside the event horizon (4.29). The largest value of $r_{s+}(\theta)$ is $2 D$, so this singularity will be naked unless

$$
2 D<M_{0}+\sqrt{M_{0}^{2}-a_{0}^{2}}, \quad \text { or } \quad \sqrt{M_{0}^{2}-a_{0}^{2}}>-M_{0} \cos (2 \alpha) .
$$


This is always satisfied if $\cos (2 \alpha)>0(M>D)$. In that case, the spacetime of metric (5.20) is a regular black hole for $\left|a_{0}\right| \leq M_{0}=M+D$, extremality being achieved for $\left|a_{0}\right|=M+D$. If $\cos (2 \alpha)<0(M<D)$, the condition for black hole regularity is stronger:

$$
\left|a_{0}\right|<\sqrt{2}|Q| .
$$

In particular, the value $\alpha=\pi / 2$ leads from (5.19) to the massless, neutral solution

$$
\begin{aligned}
\mathrm{d} s^{2} & =\mathrm{d} t-\frac{g_{0}}{\Delta_{0}} \mathrm{~d} r^{2}-g_{0} \mathrm{~d} \theta^{2}-\Delta_{0} \sin ^{2} \theta \mathrm{d} \varphi^{2}, \\
A & =0, \quad \mathrm{e}^{2 \phi}=1+\frac{2 D r}{g_{0}}, \quad \kappa=-\frac{2 a_{0} D \cos \theta}{\Sigma_{0}},
\end{aligned}
$$

with a static metric but non-vanishing axionic dipole charge $a_{0} D$. This solution is singular $\left(g_{0}=\Sigma\right.$ vanishes for $\left.r=r_{s+}(\theta)>r_{h+}\right)$, including in the static $\left(a_{0}=0\right)$ case.

\section{Conclusion}

In this paper, we first constructed static multicenter solutions of phantom EMD. We found that these lead to regular black holes in two cases. The first case is that of EMD (phantom Maxwell and dilaton fields), for the discrete values $\lambda^{2}=(p+2) / p$ ( $p$ positive integer) of the dilaton coupling constant. In this case the singular centers are hidden behind the horizon of order $p$. The number of connected components of this horizon may be equal to, or smaller than, the number $n$ (unrelated to $p$ ) of the centers. In particular, the integration constants can be arranged so that the solution represents a black hole without spatial symmetry, with a single horizon component hiding the $n$ singularities. The second case corresponds to $\mathrm{EM} \overline{\mathrm{D}}$ (phantom dilaton field), for the discrete values $\lambda^{2}=(p-2) / p(p>2$ integer). In this case, which generalizes the Majumdar-Papapetrou multi-black holes of Einstein-Maxwell theory, each center corresponds to a connected component of the event horizon. All these multicenter black holes have an infinite horizon area and vanishing temperature.

We then considered sigma models for the special values of the dilatonic coupling constant $\lambda=0, \lambda=-1$ and $\lambda=-\sqrt{3}$, for which stationary phantom EMD (or EMDA in the case $\lambda=-1$ ) reduced to three dimensions has a symmetric target space $G / H$. This occurs only for a non-phantom (or vanishing) dilaton field, so that these sigma models do not admit multicenter solutions. In all cases, the noncompact isotropy subgroup $H_{+}$ corresponds to the normal case and the compact isotropy subgroup $H_{-}$corresponds to the phantom case. This can be understood if one notes that changing the sign of the gravitational coupling constant $\eta_{2}$ of the Maxwell field is equivalent to changing the sign of the spacetime metric. Thus reduction of the four-dimensional phantom theory relative to a timelike Killing vector is equivalent to reduction of the normal theory relative to a spacelike Killing vector, which is well-known [9] to lead to a compact isotropy subgroup.

We also in each case applied a particular sigma-model transformation to generate phantom static and rotating charged solutions from a neutral solution. While the charging transformation in the normal case belongs to an $S O(1,1)$ subgroup of $H_{+}$, it belongs in the phantom case to an $S O(2)$ subgroup of $H_{-}$, resulting in important differences between the two cases. For the normal theories, an infinite $S O(1,1)$ boost leads, in the static case, to an extreme black hole saturating the lower bound $M^{2} \geq Q^{2} /\left(1+\lambda^{2}\right)$ [16]. For the phantom theories there is, for $\lambda^{2} \leq 1$, no such lower bound and massless solutions are possible [5], as obtained here in the cases of EM and $\mathrm{E} \overline{\mathrm{M}} \mathrm{DA}$ for an $S O(2)$ rotation of $\pi / 2$. Moreover, the extreme solution, obtained by a rotation of $\pi$ in the case of $E \bar{M}$, or by a rotation of $\pi / 2$ in the cases of phantom Kaluza-Klein theory or of $E \bar{M} D A$, is singular.

We have seen that, in the case of phantom Kaluza-Klein theory, the naked singularity of this static extreme solution, or more generally that of a sector of the family of phantom rotating charged solutions, becomes a mere coordinate singularity once the four-dimensional solution is lifted to five dimensions. By analogy with 
this, and with similar mechanisms of higher-dimensional resolution of naked curvature singularities [27], one can speculate that the naked singularities of a sector of the family of solutions (5.20) of EMDA could be resolved by lifting the theory to some higher-dimensional theory.

Acknowledgement: M.E.R. and J.C.F. thank CNPq (Brazil) for partial financial support.

\section{References}

[1] N. Arkani-Hamed, H.-Ch. Cheng, M.A. Luty and S. Mukohyama, JHEP 0405, 074 (2004) (arXiv:hep-th/0312099)

[2] E. Komatsu et al., Astrophys. J. Suppl. 192, 18 (2011) (arXiv:1001.4538 astro-ph])

[3] Ch. Lin, R.H. Brandenberger, L.P. Levasseur, A Matter bounce by means of ghost condensation (arXiv:1007.2654[hep-th])

[4] H.G. Ellis, Journ. Math. Phys. 14, 104 (1973); K. Bronnikov, Acta Phys. Pol. B 4, 251 (1973)

[5] G.W. Gibbons and D.A. Rasheed, Nucl. Phys. B 476, 515 (1996) (arXiv:hep-th/9604177)

[6] G. Clément and A. Fabbri, Class. Quantum Grav. 16, 323 (1999) (arXiv:gr-qc/9804050)

[7] K.A. Bronnikov, G. Clément, C.P. Constantinidis and J.C. Fabris, Phys. Lett. A 243, 121 (1998) (arXiv:gr-qc/9801050); Grav. \& Cosm. 4, 128 (1998) (arXiv:gr-qc/9804064); K.A. Bronnikov, C.P. Constantinidis, R.L. Evangelista and J.C. Fabris, Int. J. Mod. Phys. D 8, 481 (1999) (arXiv:gr-qc/9804064)

[8] G. Clément, J.C. Fabris and M.E. Rodrigues, Phys. Rev. D 79, 064021 (2009) (arXiv:0901.4543 hep-th])

[9] P. Breitenlohner, D. Maison and G.W. Gibbons: Commun. Math. Phys. 120, 295 (1988)

[10] H. Stephani, D. Kramer, M. MacCallum, C. Hoenselaers and E. Herlt, Exact Solutions to Einstein's Field Equations $2^{\text {nd }}$ Edition, Cambridge University Press, Cambridge (2003)

[11] A. Papapetrou, Proc. Roy. Irish Acad. A 51, 191 (1947); S.D. Majumdar, Phys. Rev. 72, 390 (1947)

[12] G. Neugebauer, Habilitationsschrift, FSU Jena (1969); Z. Perjès, Phys. Rev. Lett. 271668 (1971); W. Israel and G.A. Wilson, Journ. Math. Phys. 13865 (1972)

[13] G. Clément, Gen. Relat. Grav. 18, 861 (1986); Phys. Lett. A 118, 11 (1986)

[14] G. Clément and D. Gal’tsov, Phys. Rev. D 54, 6136 (1996) (arXiv:hep-th/9607043)

[15] D.V. Gal'tsov, A.A. García and O.V. Kechkin, Class. Quantum Grav. 12, 2887 (1995) (arXiv:hep-th/9504155)

[16] G.W. Gibbons, D. Kastor, L.A.J. London, P.K. Townsend and J. Traschen, Nucl. Phys. B 416, 850 (1994) (arXiv:hep-th/9310118)

[17] G. Clément and C. Leygnac, Phys. Rev. D 70, 084018 (2004) (arXiv:gr-qc/0405034)

[18] D.V. Gal'tsov and O.V. Kechkin, Phys. Rev. D 54, 1656 (1996) (arXiv:hep-th/9507005)

[19] F.J. Ernst, Phys. Rev. 167, 1175 (1968); 168, 1415 (1968) 
[20] W. Kinnersley, Journ. Math. Phys. 14, 651 (1973)

[21] G. Clément, Phys. Rev. D 57, 4885 (1998) (arXiv:gr-qc/9710109)

[22] D. Maison, Gen. Relat. Grav. 10, 717 (1979)

[23] D. Rasheed, Nucl. Phys. B 454, 379 (1995) (arXiv:hep-th/9505038)

[24] R. Gilmore, Lie groups, Lie algebras, and some of their applications, John Wiley \& Sons, New York (1974)

[25] M. Azreg-Aïnou, G. Clément and D.V. Gal'tsov, in preparation

[26] A. Sen, Phys. Rev. Lett. 69, 1006 (1992) (arXiv:hep-th/9204046)

[27] G.W. Gibbons, G.T. Horowitz and P.K. Townsend, Class. Quantum Grav. 12, 297 (1995) (arXiv:hep-th/9410073) 